\title{
Erratum to: Urinary antihypertensive drug metabolite screening using molecular networking coupled to high-resolution mass spectrometry fragmentation
}

\author{
Justin J. J. van der Hooft ${ }^{1}$ - Sandosh Padmanabhan ${ }^{2} \cdot$ Karl E. V. Burgess ${ }^{1}$ • \\ Michael P. Barrett ${ }^{1,3}$
}

Published online: 30 July 2016

(C) Springer Science+Business Media New York 2016

\section{Erratum to: Metabolomics (2016) 12:125 \\ DOI 10.1007/s11306-016-1064-z}

The original version of this article unfortunately contained a mistake. In electronic supplementary material the information about "Raw data file" was missing. This is now corrected through this erratum.

Raw data files: Raw spectral data and mzXML files are available at MetaboLights: http://www.ebi.ac.uk/metabo lights/MTBLS307.

Electronic supplementary material The online version of this article (doi:10.1007/s11306-016-1078-6) contains supplementary material, which is available to authorized users.

The online version of the original article can be found under doi: 10.1007/s11306-016-1064-z.

Justin J. J. van der Hooft

justin.vanderhooft@glasgow.ac.uk

1 Glasgow Polyomics, College of Medical, Veterinary and Life Sciences, University of Glasgow, Glasgow, UK

2 Institute of Cardiovascular and Medical Sciences, College of Medical, Veterinary and Life Sciences, University of Glasgow, Glasgow, UK

3 Wellcome Trust Centre for Molecular Parasitology, Institute of Infection, Immunity and Inflammation, College of Medical, Veterinary and Life Sciences, University of Glasgow, Glasgow, UK 